\title{
outlook
}

\section{Michael Fischbach: Homing in on the molecules from microbes}

Michael Fischbach, a bioengineer at Stanford University in California, describes the small molecules produced by microbes in the gut as a surprisingly unexamined field. To explore it, his team has developed a way to 'toggle' the molecules on and off to find out exactly what they do.

How is your work helping you to investigate the molecules made by gut microbes? It gives us a way to study the effects of molecules produced by the gut microbiome, one molecule at a time. We developed our genetic system for a commensal strain of Clostridium that produces a lot of molecules that are characteristic of the microbiome. They are made in very high numbers and they show up in the host's bloodstream, but there was no good way of working out what they do. Our genetic system can stop the production of specific molecules; when we colonize mice with a mutant bacterial strain, the mice are deficient in those molecules. We then compare them with mice colonized by the wild-type bacterial strain and that do have the molecules, and look for differences.

What type of molecules are you studying? We are focusing on branched, short-chain fatty acids. These are chemically similar to acetate, propionate and butyrate - well-studied short-chain fatty acids from commensal bacteria. But the ones we are looking at are produced by a different metabolic routethey come from branched-chain amino acids and not much is known about them.

\section{Why did you focus on these fatty acids?}

We are trying to look at molecules that are produced in great abundance. They are naturally present in the body at concentrations similar to those of therapeutic drugs. So there is no question about whether these molecules end up permeating the host - they do. There are dozens of them, and we feel it is warranted to take the time to treat these molecules almost like a classic pharmacology problem: you have a molecule and you want to know everything about what it does. So we try to find out what tissues it acts in, what receptors it interacts with and how that brings about an effect on host biology. It's really fundamental stuff that I think is going to end up in textbooks one day.

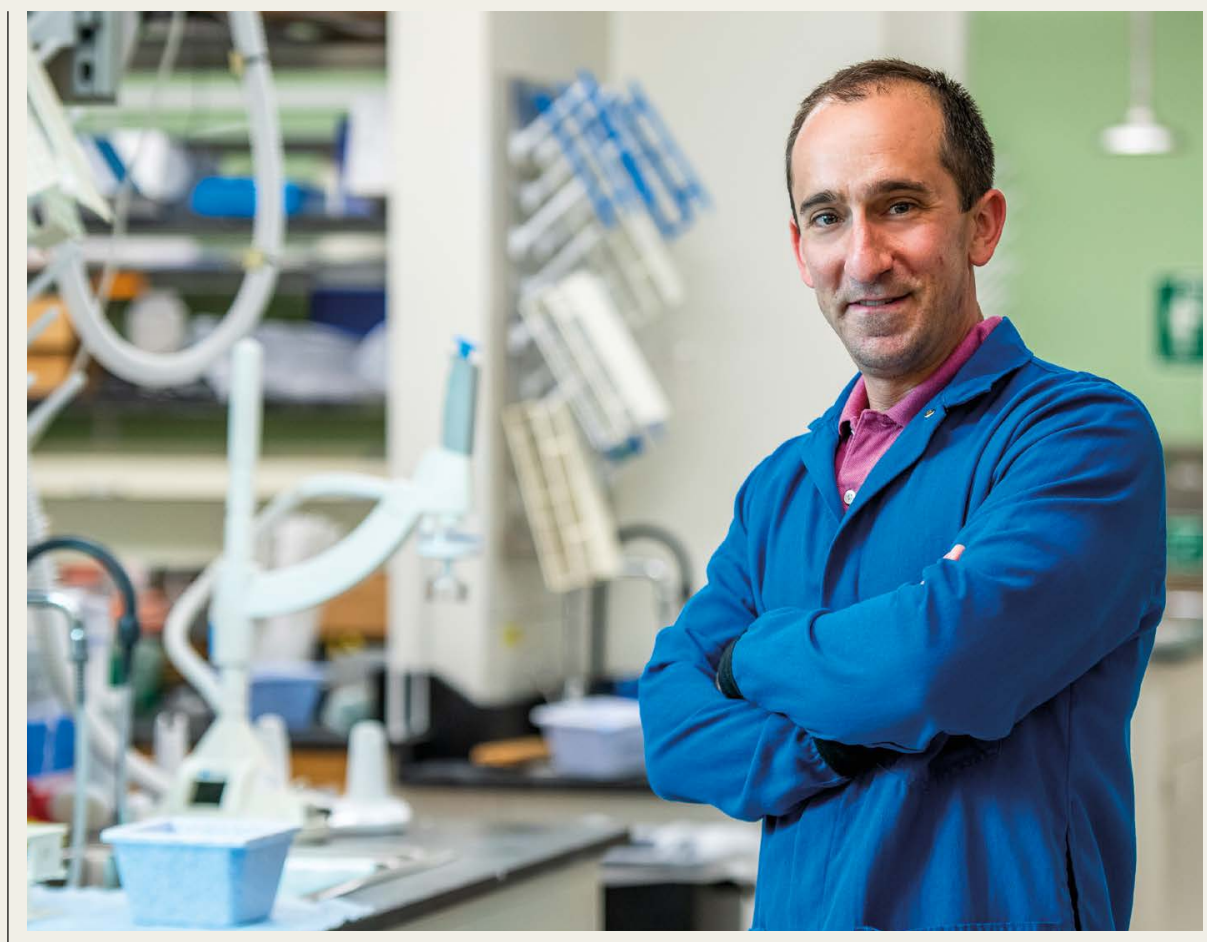

Michael Fischbach investigates the effects of molecules produced by gut microbes.

What have you caught the molecules doing? We are finding a range of immunological differences that can be attributed to the presence or absence of individual molecules produced by microbes. For example, the branched, shortchain fatty acids seem to modulate the activity of cells that produce the protein immunoglobulin $\mathrm{A}$, which is involved in barrier protection in mucous membranes. We don't have the complete story yet, but we are starting to work out the mechanism of that effect. My guess is that, in addition to modulating the immune system, the molecules do other things too. We just haven't had time to explore it all yet.

Could microbial molecules lead to drugs that will replace whole microbe probiotics?

Both options are on the table. If we can identify the receptors that microbial molecules bind to, we might be able to design drugs that interact with those receptors. We could mimic the effects microbial molecules have on the receptors, or we might want to block their effects. Equally, we could also use the microbes themselves. The use of whole microbes could mean delivering the effects of several molecules at a time, and could ensure more lasting effects.
There is little precedent for using microbes as drugs, and doing so will bring regulatory challenges. But we will probably see both approaches moving forward. And both could be very powerful.

Where do you see this kind of work heading? We have used bacterial genetics to create a clean system for comparing two groups of mice that differ only in the presence or absence of a microbiome-derived molecule. But we did this in a rather unnatural setting: a mouse with only one bacterial species in its gut. Now, the biggest need is to create a model system for experiments such as these: bacterial communities that are completely defined, but complex enough to be similar to the native gut community. Using a community that complex, we could carry out experiments in which we remove one bacterial species - or even one gene from one bacterial species. Those are more likely to stand the test of time.

\section{Interview by Andrew Scott}

This interview has been edited for length and clarity. 